\title{
Significance of the Nested Structure in Multiplex World Trade Networks
}

\author{
Zhuo-Ming Ren $\mathbb{D D}^{1}{ }^{1}$ Xiao Pan, ${ }^{1}$ and Yi-Cheng Zhang ${ }^{2}$ \\ ${ }^{1}$ Alibaba Business School, Hangzhou Normal University, Hangzhou 311121, China \\ ${ }^{2}$ Department of Physics, University of Fribourg, Fribourg 1700, Switzerland \\ Correspondence should be addressed to Zhuo-Ming Ren; zhuomingren@gmail.com
}

Received 10 August 2020; Revised 10 November 2020; Accepted 27 November 2020; Published 9 December 2020

Academic Editor: Y. T. Peter Bian

Copyright (C) 2020 Zhuo-Ming Ren et al. This is an open access article distributed under the Creative Commons Attribution License, which permits unrestricted use, distribution, and reproduction in any medium, provided the original work is properly cited.

\begin{abstract}
The hierarchically nested structure is widely observed in a broad range of real systems, encompassing ecological networks, economic and trade networks, communication networks, among many others. However, there remain statistical challenges of the prevalence of nestedness. In response to this problem, we focus on the effect of incomplete information and the inputted matrix size, the role of network density and degree sequences, and the relevance of degree-degree correlation to conduct systematic research on the significance of the nested structure according to multiplex world trade networks. Firstly, the nested structure can observe significantly when suffering incomplete information and varying inputted matrix size. Secondly, to analyze the role of network density and degree sequences in nested structure, we use "swappable rows, swappable columns" null model which conserves the network size and density, and "fixed in-degree, fixed out-degree" null model which not only preserves the network size and density but also keeps node degree to do randomization tests of nestedness. The randomizations of two null models remark that most nested structures are not determined by network density and degree sequences but closely related to them. Finally, we investigate degree-degree correlation in nested networks, and the results show that the nested structure is negatively related with degree-degree correlation of the network. Following the empirical analysis train of thought, we argue that nestedness is still a unique feature of the network.
\end{abstract}

\section{Introduction}

The nested structure has been observed in ecological and socioeconomic systems as diverse as ecological mutualistic organizations, world trade, and interorganizational relations, among many others $[1,2]$. Given a network of interacting nodes, nested structure can be described as the tendency for nodes to interact with subsets of the interaction partners of better-connected nodes. A perfectly nested matrix can be shown that the entries in each successive row and column are respectively a strict subset of those in the previous row and column [3]. In ecological systems, nested patterns in mutualistic networks are still open challenges that have led to multiple debates to date such as biodiversity and structural stability [4-7]. In economic systems, one has often access to only limited information about the nested structure of the social and economic networks to capture economic complexity $[8,9]$. The nested feature has already inspired a number of methods quantifying different aspects of socioeconomic systems which include exploring the nested nature of the observed networks $[1,10]$, as well as predicting the evolution of industrial ecosystems [11].

Although scholars are currently debating around the role of the nested structure in ecological and economic systems, the observed architecture of ecological and socioeconomic networks differs significantly from that of random networks [12]. A lot of works are fascinated by statistical challenges in null model analysis to uncover the network structure $[13,14]$. For instance, Strona et al. [15] developed a new procedure named the curveball algorithm, in that it focused rather on matrix information content than on matrix structure. Fosdick et al. [16] investigated the configuring random graph models with fixed degree sequences. Orsini et al. [17] gave the release solution to the evaluation of 
network randomness, and the null model analysis was used to confirm the prevalence of nestedness. Jonsson [18] analyzed randomization tests of nestedness in 52 mutualistic networks and showed that they were highly nested, and nestedness increased with the number of interactions of the network. Staniczenko et al. [4] showed that complex ecological networks were binary nested, but quantitative preferences were nonnested. Payratoborras et al. [19] applied a theoretical approach to the analysis of 167 real mutualistic networks that nestedness was not an irreducibly macroscopic feature but an entropic consequence of the degree sequences. Furthermore, the similar efforts could try to distinguish nestedness and network property such as modularity [20, 21], core-periphery structure [22], and degree-degree correlation [23]. These studies are trying to solve statistical challenges of the prevalence of nestedness, but the networks that they used are different types or through multiple channels. Naturally, the network structure of world trade networks is complex [24, 25]. Although the scale of the networks is limited by the number of countries and the number of products, not all countries participate in trade which ultimately leads to different network sizes for different products. Therefore, these networks are not the same size and scale, and other network characteristics are also different (as shown in Figure 3). So, the multiplex world trade network is a good sample for analyzing the significance of nested structure. Recently, the network structure can be significantly investigated in the worldwide trade network [26] or be extended to multilayer network [27]. There have also been many recent studies on the null model or the prevalence of nestedness in world trade networks. For instance, the randomization method can assess in detail the role patterns of the world trade networks in binary [28] or weighted [29], monopartite or bipartite [30], aggregated or disaggregated by commodity [31], or across several years [32].

In this paper, we remark the significance of nested structure according to hundreds of world trade networks. The statistical challenges of the prevalence of nestedness are mainly solved from the following three aspects: firstly, how is the robustness of the nested structure when suffering incomplete information and varying the inputted matrix size. Secondly, to analyze the role of network density and degree sequences in nested structure, we will use two null models to do randomization tests of nestedness. Finally, we will investigate the relationships between nestedness and four types of the degree-degree correlation in multiplex world trade networks.

\section{Methods and Materials}

Figure 1 illustrates the adjacency matrices of three toy networks with the same number of elements but the different level of nestedness. The network visualizations of these three matrices are given in Figure 1(a). Although the number of nodes and links are the same in these networks, the level of nestedness is totally different as the nestedness is determined by the particular directionality of links as revealed if the rows and columns of these matrices are both reordered by descending degree $(k)$ in Figure 1(b). The matrix with the triangular structure on the right is fully nested, while the matrix with random shape on the left is not nested.

Generally, we can define that the network consists of sets of generalist nodes and sets of specialist nodes. The specialists interact only with a small subset of generalist nodes, while the generalists interact with (almost) all other nodes in the network. This network organization reveals the nested structure. Nestedness is a statistical property of interaction data presented in matrix form. Here, a simple and widely used metric is named the Nested Overlap and Decreasing Fill (NODF) [33]. In the beginning of calculation, the row and the column of a matrix $\left(A_{m * n}=\left\{a_{i j}\right\}\right)$ are swapped and rank-ordered by the sum of the presences in each of these rows and columns, respectively. The transformed matrices are then ready to be processed by the following equation:

$$
\begin{gathered}
\eta_{i j}=\sum_{i<j} \begin{cases}0, & \text { if } k_{i}=k_{j}, \\
\frac{\sum_{l} a_{i l} a_{j l}}{\min \left(k_{i}, k_{j}\right)}, & \text { otherwise, }\end{cases} \\
\eta=\frac{\sum_{i<j}\left(\eta_{i j}^{\text {row }}+\eta_{i j}^{\text {column }}\right)}{(n(n-1) / 2)+(m(m-1) / 2)} .
\end{gathered}
$$

Here, $k_{i}$ is the number of 1 in $i^{\text {th }}$ row, i.e., degree. The $\eta$ takes values between 0 (unnested) and 1 (perfectly nested). Supplementary Information S1 describes the step by step calculation of the nestedness measurement (NODF), and Figure S1 gives an example of calculation of nestedness which is from ref [3]. According to the NODF metric, the nestedness of three toy networks, respectively, corresponds to $0,0.5$, and 1 , as shown in Figure 1.

To further analyze the characteristics of nested structure, we use the world trade datasets to construct commodity networks as analysis objects. These datasets consist of imports and exports both by destination. The data comprises 261 countries and 786 products (categories). The detailed introduction of the data is seen in Supplementary Information S2 and Figure S2. For a target product p, we can select the trade interactions just contained the target product trade information and then build a matrix $M_{c c^{\prime}}^{p}=\left\{v_{c^{\prime}}^{p}\right\}$, which can be expressed as the country $c$ exports the target product $p$ to the country $c^{\prime}$. To build a binary network, it is necessary to check whether a trade interaction performance revealed comparative advantage [34]. We need to estimate whether a trade interaction between two countries is significant in the trade network of the given product and can calculate whether a trade interaction's share of a country's trade is larger or smaller than the country's share of the entire whole market. Mathematically,

$$
\operatorname{RCA}_{\mathrm{cc}^{\prime}}^{p}=\frac{v_{\mathrm{cc}^{\prime}}^{p} / \sum_{c} v_{\mathrm{cc}^{\prime}}^{p}}{\sum_{c^{\prime}} v_{\mathrm{cc}}^{p} / \sum_{c} \sum_{c^{\prime}} v_{\mathrm{cc}^{\prime}}^{p}},
$$

where $v_{\mathrm{cc}^{\prime}}^{p}$ is equal to the dollar volume of a trade interaction between country $c$ and country $c^{\prime}$. The natural cutoff used to determine whether a trade interaction has revealed 

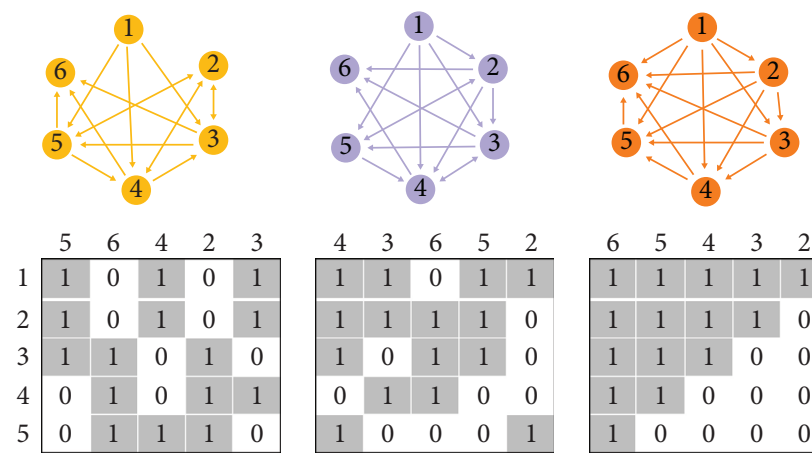

\begin{tabular}{|c|c|c|c|c|}
\hline 6 & 5 & 4 & 3 & 2 \\
\hline 1 & 1 & 1 & 1 & 1 \\
\hline 1 & 1 & 1 & 1 & 0 \\
\hline 1 & 1 & 1 & 0 & 0 \\
\hline 1 & 1 & 0 & 0 & 0 \\
\hline 1 & 0 & 0 & 0 & 0 \\
\hline
\end{tabular}

Figure 1: Three representations of matrices, respectively, correspond to none, moderate, and perfect nested network. (a) These three networks have the same number of nodes and links. (b) In each matrix, the rows and columns have been ranked by the number of ones (i.e., degree). In a perfectly nested matrix, the upper-left triangle would be full of elements and the lower-right triangle would have no elements.

comparative advantage is RCA $\geq \lambda$. We can therefore construct the binary country-country matrix from the RCA matrix, if we consider,

$$
a_{\mathrm{cc}^{\prime}}^{p}= \begin{cases}1, & \text { if } \mathrm{RCA}_{\mathrm{cc}^{\prime}}^{p} \geq \lambda, \\ 0, & \text { if } \mathrm{RCA}_{\mathrm{cc}^{p}}^{p}<\lambda .\end{cases}
$$

Thus, we can construct a directed network for a given product from view of network science. The nodes represent countries, and then if $a_{\mathrm{cc}^{\prime}}^{p}=1$, there is a link connected between two countries $c$ and $c^{\prime}$. The same steps for each product; we can build 786 networks. It is emphasized that we only remain each network of countries which are more than ten (i.e., the number of nodes is more than ten). We investigate the relevant characteristics of the multiplex world trade network as shown in Figure 2. We can see that the matrix size, network density, and average degree in hundreds of networks are extensive. From Figure 2(a), we find that the inputted matrix size is mainly distributed between 15000 and 45000, and in Figure 2(b), the network density is mainly around 0.06 and 0.1 , then the percentage gradually decreases after being greater than 0.1. Further, the statistics of the average degree of the network is shown in Figure 2(c); we can observe that the average degree of the network is mostly around 35 to 45 . The network size, network density, and network degree of each layer of the multilayer network are different. So, the multiplex world trade network is a good sample for analyzing the significance of nested structure.

After inputting matrices, we can quantify the nestedness in each layer of the multilayer network with the NODF metric and report their distribution in Figure 3. We set $\lambda=$ 0.1 and the nestedness distributions under other threshold as qualitatively similar (the effect of $\lambda$ on results is shown in Figures S3, S4, and S5). One can see that the nestedness of layers varies significantly. The range of nestedness values is $[0.1,0.6]$, which is close to normal distribution. In addition, the matrices are representations of the different layers of world trade networks which respectively correspond to the network of bovine, motorcycles, and medical instruments seen in Figure S6. The nestedness of bovine, motorcycles, and medical instruments is $0.12,0.37$, and 0.57 respectively, which locates in the low, medium, and high areas of nestedness distribution. Although the scale of the matrices are limited by the number of countries and the number of products, not all countries participate in trade which ultimately leads to different matrix sizes for different products. Therefore, these networks are not the same size and scale as toy networks. The network size, network density (the ratio between the number of ones and network size), and other network characteristics of the three networks are different which also suggests a good sample for analyzing the significance of nested structure.

\section{Results}

As previously shown by the network of bovine, motorcycles, and medical instruments, the structure of the three networks is different. We are interested in the relations between nestedness and the common network features including the network size, network density, and degree.

\subsection{The Effect of Incomplete Information and Inputted Matrix}

Size. The collected world trade data are inevitable to exist absences and some noise. For example, it is difficult to collect trade data of some countries such as Iraq and North Korea. In addition, the trade volume in the collected data could not be completely accuracy in statistics. This is practically meaningful, as in reality, the missing trading data could happen commonly [35,36], so we need to consider these two situations and employ two ways to simulate these problems. The first is that randomly swapping the interactions is limited to two products in the pairs of same countries with the exporter and the importer. We randomly choose an amount of trade interactions and independently run the swapping simulations. The swapping ratio is between the chosen interactions and the total interactions. The second is randomly removing a certain amount of trade interactions. The removing ratio is between a certain amount of removed trade interactions and the total interaction. We then investigate whether the nestedness ranking is influenced significantly in the case of incomplete and noisy information and compute Kendall's tau correlation coefficient [37] between the original nestedness list of products and their new nestedness list. As revealed in Figure 4(a), the lower 


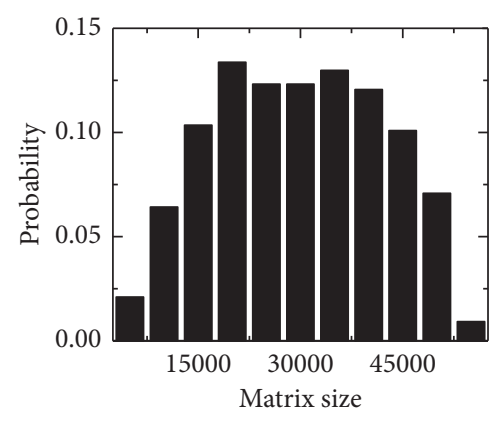

(a)

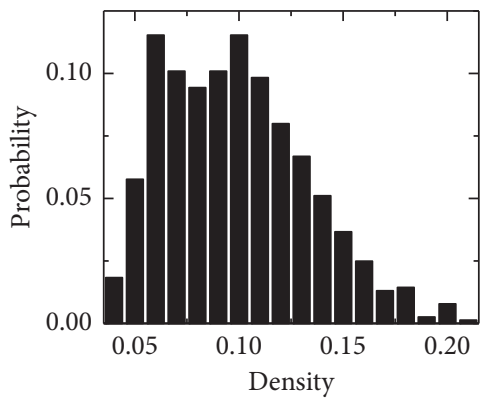

(b)

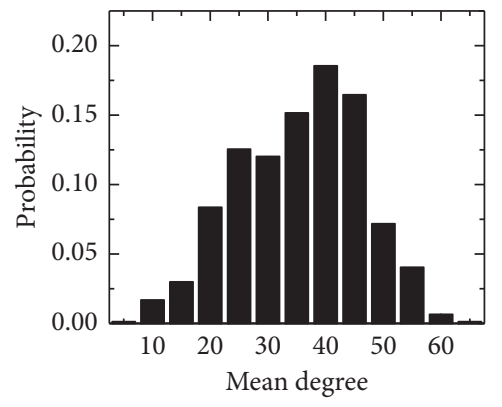

(c)

Figure 2: The relevant characteristics of the multiplex world trade network. (a) The distribution of the matrix size corresponding to the multiplex world trade network. The matrix size of each network is that the number of rows multiplies the number of columns. (b) The distribution of the network density (the ratio between the number of ones and matrix size). (c) The distribution of network average degree (i.e., the mean of in-degree and out-degree).
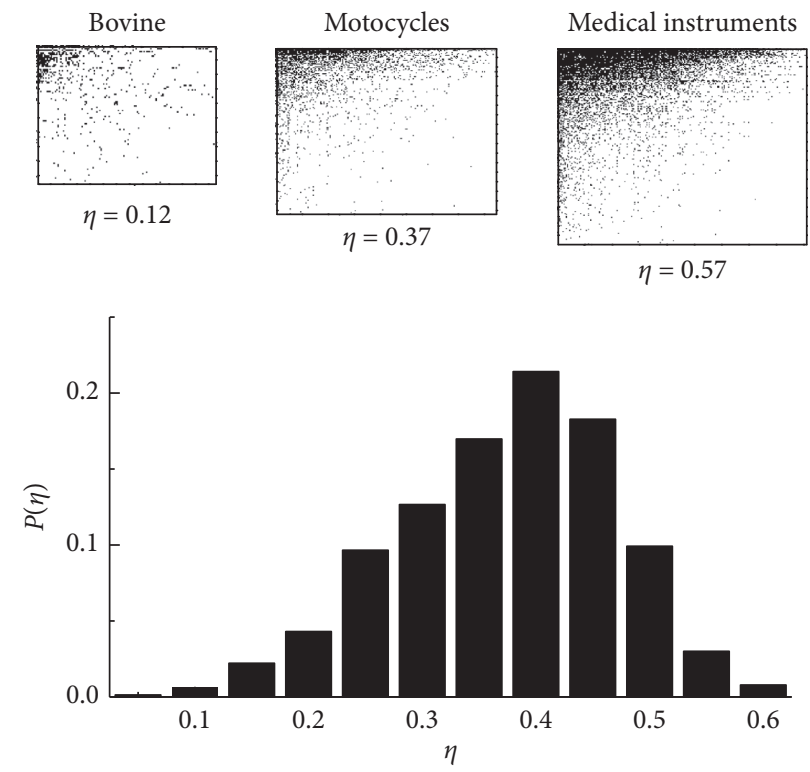

FiguRE 3: Nested structure in world trade networks. (a) The three matrices are representations of the different layers of world trade networks which respectively correspond to the network of bovine, motorcycles, and medical instruments. The shape of these three matrices (i.e., the number of rows and columns) is $136 * 96,212 * 174$, and $228 * 210$. The nestedness of matrices $(\eta)$ is $0.12,0.37$, and 0.57 , respectively. (b) The curve is the distribution of nestedness calculated by all matrices of products.

correlation value is that the larger the link swapping ratio and link removing ratio are, the correlation just drops a little. It indicates that the nested structure is robust against incomplete and noisy information. We can also find that when links have changed, the nested structure of whole trade networks after removing links is more robust than that after swapping links.

The other problem is that some countries do not have data or different thresholds of RCA, resulting in different sizes of the inputted matrix. So, we analyze the impact of the size of the matrix on the nestedness of the each network. It has already been pointed out that in sparse networks, the high nestedness might not result from the significant triangular pattern in the adjacency matrix, but due to the large number of empty rows and columns $[6,23,38]$. In these cases, the nestedness metric $\eta$ will become much smaller if these empty rows and columns are removed. To examine whether the nested structure in the multilayer trading networks is truly significant, in each layer, we one by one remove the remaining nodes with the smallest degree. We then compute Kendall's tau rank correlation between original nestedness of layers and their new nestedness list after a fraction of smallest degree nodes are removed. The detailed process is as follows. We first introduced basic two definitions. Network size is defined as the length of column multiplied by the length of row in inputted matrix. The given network has $M$ links, and then network density is the total number of links divided by network size. For each inputted matrix, the size of matrix is different. We investigate effects of network size on measuring nestedness. The transformed matrices are then ready to be processed by the nestedness metric, which is that the rows and the columns of a matrix are swapped and rank-ordered by the sum of the presences in each of these rows and columns, respectively. After this, 


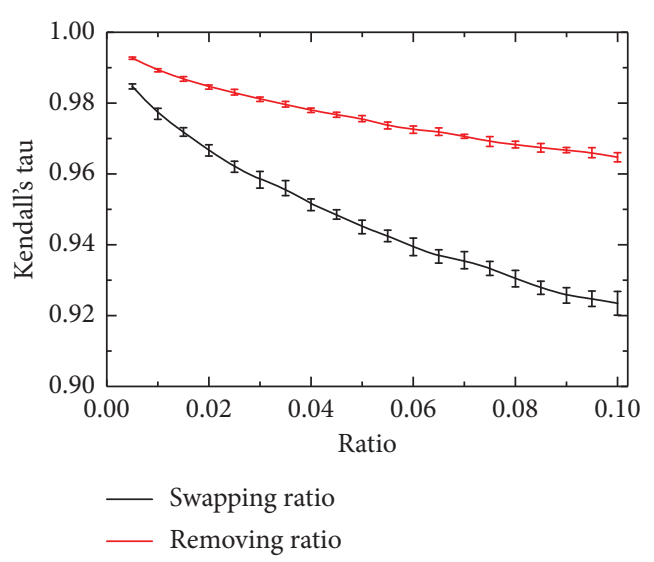

(a)

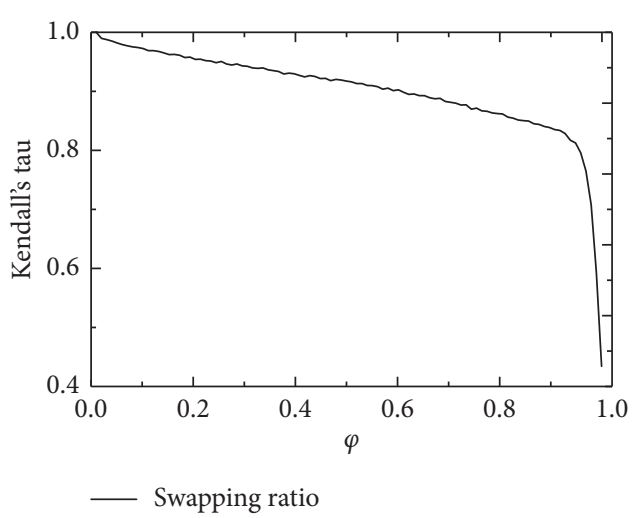

(b)

FIgURE 4: The effect of incomplete information and inputted matrix size. (a) The significance of nested structure under incomplete information. (b) The significance of nestedness in the varied structured triangle.

the rows and columns are closed to the border of the matrix, which are very sparse. We first remove a certain number $l$ of rows and columns from the margin of a triangular matrix defined as $A_{m n}$. Then, we calculate the nestedness of the resulting removed matrix $A_{(m-l)(n-l)}$ and the removal ratio

$$
\varphi=1-\frac{(m-l)(n-l)}{m * n} .
$$

Finally, the Kendall rank correlation is employed to analyze the relation between original nestedness list and new nestedness list. In Figure 4(b), one can see that as the removal ratio increases, the correlation gradually decreases, indicating that as the matrix size changes, the nestedness list of the networks also changes, but the correlation can reach 0.8 even if $80 \%$ matrix is removed. Until $80 \%$ matrix is removed, the correlation drops sharply.

\subsection{The Relations with Network Density and Degree Sequence.} Besides network size, what is the relation with network density and degree? We will use in the entire trading networks with a variety of nested structure features to investigate relationship with statistical network density and degree, as shown in Figures 5(a) and 5(b). We find that not only network density but also network average degree (i.e., the mean of in-degree and out-degree) are all positively related to nestedness. That is to say, the larger the density of the network is, the stronger the nestedness of the network will be. The higher the average degree of the network is, the stronger the nestedness of the network will be.

Does that mean that nestedness can be expressed in terms of density and average degree? The significance of the nested pattern of the multilayer trading network is further examined by comparing it with two null models [38]. Here, the two null models (detailed introduction is also seen in Supplementary Information S4 and Figures S7 and S8). In the first one, the "swappable rows, swappable columns" (SS) null model conserves matrix dimensions (numbers of rows and columns) and fill. It works by shuffling elements at random within the matrix; however, it differs in that degenerating matrices (those containing rows/columns with no connections) are not permitted. In the second one, the "fixed in-degree, fixed out-degree" (FF) null model has subsequently been a popular choice for application to the nested networks, which preserves the degree distribution of the original network. The two links are randomly rewired but preserve the degree of the involved four nodes. This null model not only conserves the network density but also fixes degree of each node. After independently repeating 100 times of randomization for each commodity network, we adopt $z$-score which is calculated by comparing to the nestedness scores of randomizations of the network. $z-$ score $=\left(\eta-\left\langle\eta_{\text {null }}\right\rangle\right) / \sigma_{\text {null }}$, where $\left\langle\eta_{\text {null }}\right\rangle$ and $\sigma_{\text {null }}$ are, respectively, the mean and standard deviation of nestedness across a sample of randomizations of the network.

We can observe the $z$-score in Figures 5(c) and 5(d). The result of the SS model showed that the greater the nestedness is, the larger the $z$ value will be. It also reveals that $z$ value of extremely rare networks is closed to zero. These networks can be generated by SS model but their nested structure is likely to break. When the nested structure is obvious and $z$ value is also high, the network generated by SS model cannot generate the nested structure same as the real network. However, it is different in the FF model, as shown in Figure 5(d). We can find that the $z$ value of a few networks is close to 0 , that is to say, the FF model can obtain the same nested structure as the original network. But most of the $z$ value is far greater than 0 , indicating that the FF model cannot generate the same nestedness as the original network generally. In addition, overall, the greater the nestedness is, the larger the $z$ value will be, but the trend is weaker. In summary, the results indicate that degrees may determine the nested structure of a few networks, but the nestedness is not statistically determined by the network density and degree sequence.

3.3. The Relevance of Degree-Degree Correlations. Finally, we further expand the relationship between degree-degree correlations and nestedness. Let us first look at the definition 


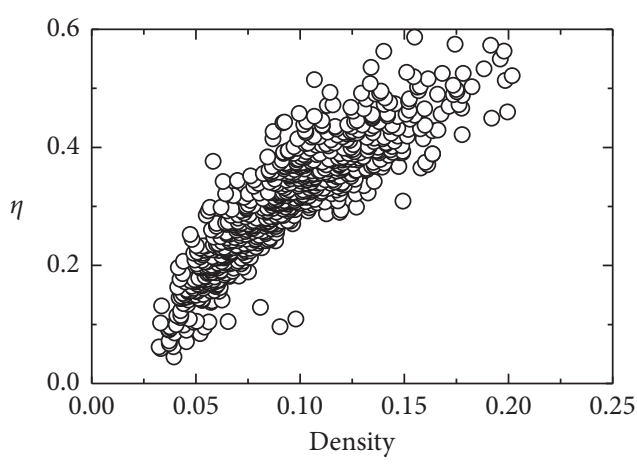

(a)

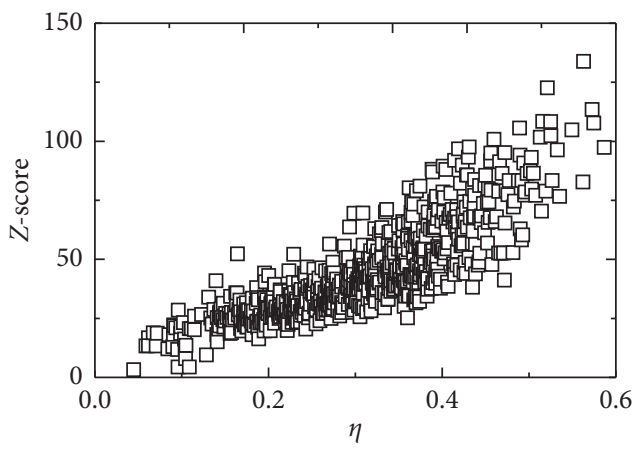

(c)

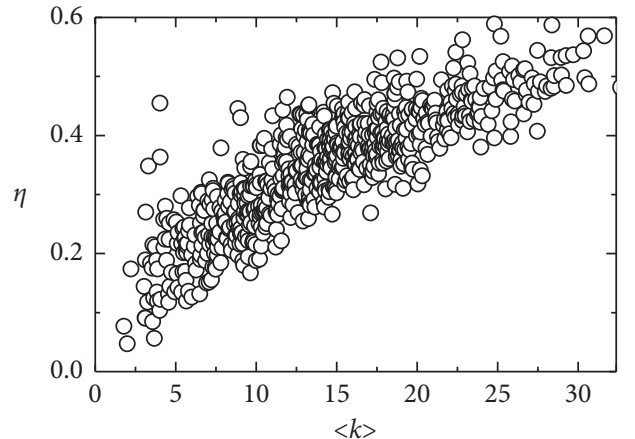

(b)

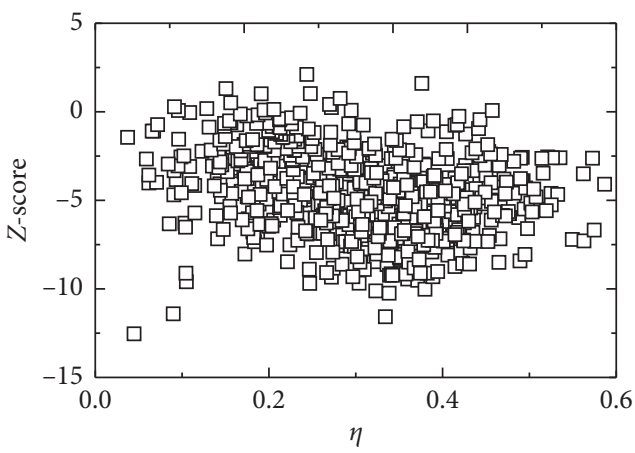

(d)

FIgURE 5: The relations with network density and degree sequence. (a) The relationship between density and nestedness. (b) The relationship between degree and nestedness. (c) $z$-score in the "swappable rows, swappable columns" (SS) null model. (d) $z$-score in the "fixed in-degree, fixed out-degree" (FF) null model. The higher the $z$-score value, the more different the nestedness of the original network is from the randomized network according to the null model.

of nestedness and degree-degree correlations. The degreedegree correlation is a standard tool for analyzing the network structure and has a simple interpretation $[39,40]$. Degree-degree correlation ranges from -1 to 1 . If $r>0$, high degree nodes tend to connect to other high degree nodes; otherwise, high degree nodes tend to connect to low degree nodes. A network exhibits nestedness if the neighborhood of a node is contained in the neighborhoods of the nodes with higher degrees. It is obvious that a network exhibits nestedness if the neighborhood of a node is contained in the neighborhoods of the nodes with higher degrees. The triangular structure thus is strongly correlated with negative degree-degree correlation. That is to say, if the nested structure is more obvious, the degree correlation value is more negative. Thus, according to the import and export relationship of the goods, the network has in-degree and out-degree. So, the degree-degree correlation could be of four types. We use the degree-degree correlation equation $[39,40]$ and the detailed illustrations are also seen in Figure S9:

$$
r=\frac{\sum_{i} k_{i}^{\alpha} k_{i}^{\beta}-M^{-1} \sum_{i} k_{i}^{\alpha} \sum_{i^{\prime}} k_{i^{\prime}}^{\beta}}{\sqrt{\left[\sum_{i}\left(k_{i}^{\beta}\right)^{2}-M^{-1}\left(\sum_{i} k_{i}^{\beta}\right)^{2}\right]\left[\sum_{i}\left(k_{i}^{\alpha}\right)^{2}-M^{-1}\left(\sum_{i} k_{i}^{\alpha}\right)^{2}\right]}},
$$

where let $\alpha, \beta \in\{$ in, out $\}$ index be the degree type and $k_{i}^{\alpha}$ and $k_{i}^{\beta}$ be the $\alpha$ - and $\beta$-degree of the source node and target node for the $i^{\text {th }}$ edge. $M$ is the number of links. Figure 6 shows the scatter plot of the nestedness $\eta$ versus four-type degree-degree correlation. One can see that the higher nestedness of a network indicates stronger negative degreedegree correlation, which is also confirmed in modeled scale-free nested networks [23]. It can be also seen that when the nestedness is small, a part of the network degree correlation is close to 0 or even positive. But when the nestedness becomes larger, it shows larger negative degree correlation, especially when the nestedness is higher than 0.2 . Comparing the four types of degree correlations, we can see that the negative correlation between $r$ (in, in) and 


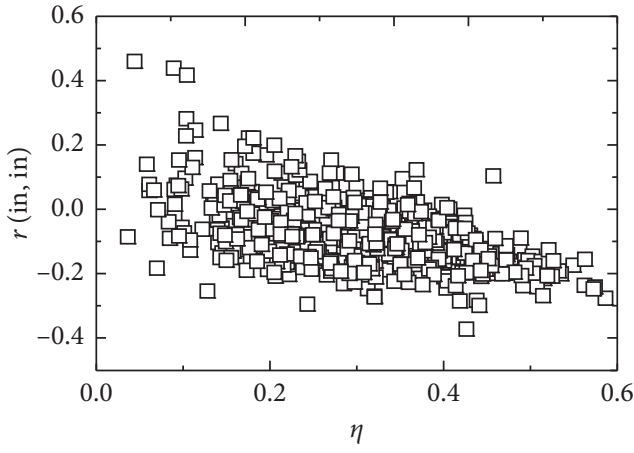

(a)

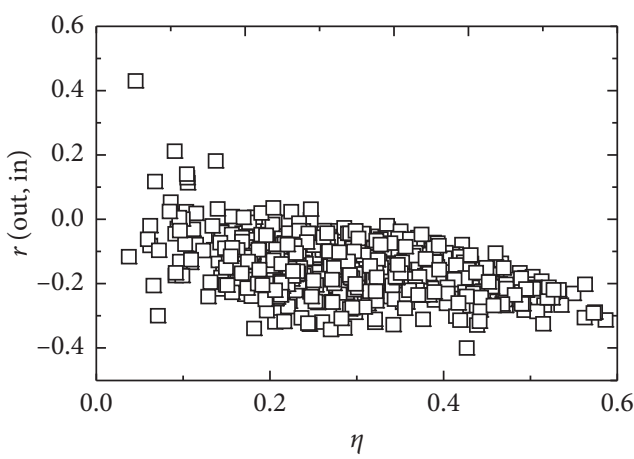

(c)

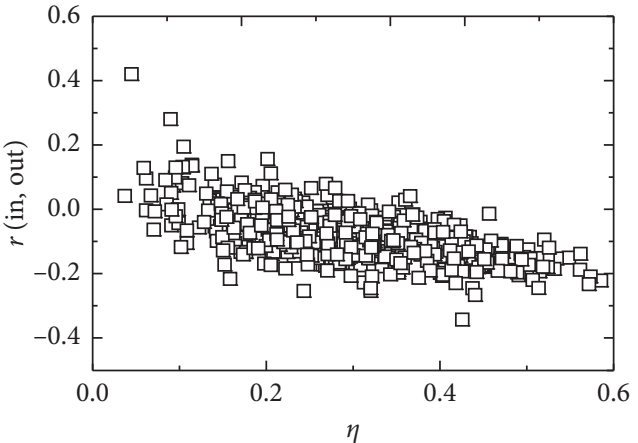

(b)

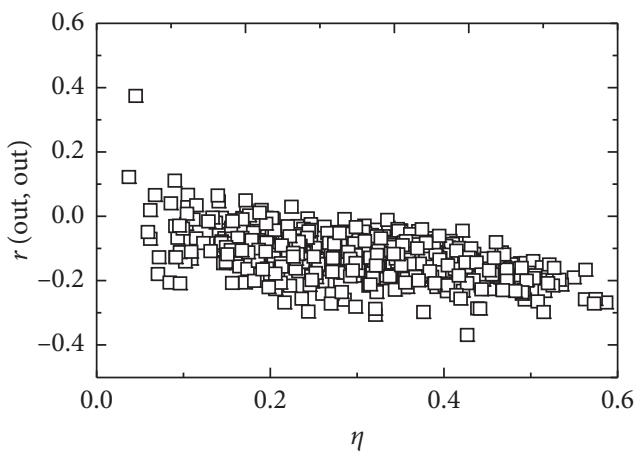

(d)

Figure 6: The four types of degree-degree correlations in nested networks. (a) $r$ (in, in) means in-degree-in-degree correlation; (b) $r$ (in, out) means in-degree-out-degree correlation; (c) $r$ (out, in) means out-degree-in-degree correlation; (d) $r$ (out, out) means out-degree-outdegree correlation.

nestedness is the most obvious, and $r$ (out, out) is the least obvious. In addition, in a network with nestedness less than $0.2, r$ (in, in) and $r$ (in, out) are more likely to be positive than $r$ (out, in) and $r$ (out, out).

\section{Conclusions and Discussion}

We conducted a systematic analysis of the significance of the nested structure in the multilayer commodity world trade networks. The analysis was from three aspects. Firstly, we simulated incomplete information and the effect of matrix size to measure nestedness. The results revealed that a small amount of data noise and network size did not affect the nested structure. Secondly, we adopted two null models to shuffle links. One could conserve the network density, the other could not only preserve the network size and density, but also keep node degree. We found that although density and degree were positively related to nested structure, they could not determine the nested structure. Finally, we further analyzed the relevance of degree-degree correlation. The results showed that the network with high nestedness has a significant negative degree-degree correlation. In summary, we believe that nested structure is closely related to the density, degree, and degree-degree correlation of the network, but nestedness is still a unique feature of the network.

Our work could enrich the statistical challenges of the prevalence of nestedness, and numerous extensions can be conducted. For instance, we mainly focused on the nestedness method named NODF, and could be asked what about the sensitivity of other nestedness detection methods [41]. In addition, we just used a commonly adopted metric to measure the nestedness of a network. How to design a better metric to capture nestedness more in social dynamics such as communication dynamics [42] is still worth studying. What's more, our work could bridge the nested structure of the world trade network and its realistic economic and management significance. As we have shown with our illustration of nested structure which can characterize the world trade network, we could bring solutions to other economic systems in observation and modeling. The nestedness could provide a way of thinking about the structural characteristics of the realistic economic systems. There also are other open questions to ask for future study. For instance, we mainly focus on the prevalence of nestedness, but what are the mechanisms that lead to the triangular nested structure of the matrix remains unclear, and a temporal analysis of the networks, in such a way to allow us for observations of how the triangular nested structure of the trade 
relations have evolved over time. They could provide a great challenge to capture the complex structure of the interactions between a large number of economic agents, which brings solutions to a variety of issues in observation, modeling, and prediction in socioeconomic systems.

\section{Data Availability}

The datasets used to support the findings of this study are available from the website (https://comtrade.un.org/) or an alternative data source (http://atlas.media.mit.edu/en/ resources/data/).

\section{Conflicts of Interest}

The authors declare that they have no conflicts of interest.

\section{Acknowledgments}

The work was partially supported by the National Natural Science Foundation of China (Grant no. 61803137), Zhejiang Provincial Natural Science Foundation of China (Grant no. LY21F030019), Qiantang River Talents Plan (Grant no. QJD1803005), and Hangzhou Normal University Research Funding Project and Foundation of High Level Overseas Returnees (Team) in Hangzhou for Pioneering Innovation Program.

\section{Supplementary Materials}

Supplementary Materials provide the following specific details. S1: the step by step calculation of the nestedness measurement (NODF) (Figure S1). S2: the basic information of the world trade datasets including the trivial details (Figure S2). S3: the detailed illustrations of the network construction. Furthermore, we also analyze the effect of the network structure corresponding to 12 different products with different RCA thresholds including network density (Figure S3), network size (Figure S4), and nestedness (Figure S5). The visualization of the network of bovine, motorcycles, and medical instruments (Figure S6). S6: the two null models to check the significant nestedness of the inputted matrix and detailed description of two null models (Figures S7 and S8). S7: the detailed illustrations of the degree-degree correlation in the nested networks (Figure S9). (Supplementary Materials)

\section{References}

[1] M. D. König, C. J. Tessone, and Y. Zenou, "Nestedness in networks: a theoretical model and some applications," Theoretical Economics, vol. 9, no. 3, pp. 695-752, 2014.

[2] M. S. Mariani, Z.-M. Ren, J. Bascompte, and C. J. Tessone, "Nestedness in complex networks: observation, emergence, and implications," Physics Reports, vol. 813, pp. 1-90, 2019.

[3] M. Almeida-Neto, P. Guimarães, P. R. Guimarães, R. D. Loyola, and W. Ulrich, "A consistent metric for nestedness analysis in ecological systems: reconciling concept and measurement," Oikos, vol. 117, no. 8, pp. 1227-1239, 2008.
[4] P. P. A. Staniczenko, J. Kopp, and S. Allesina, "The ghost of nestedness in ecological networks," Nature Communications, vol. 4, p. 1391, 2013.

[5] U. Bastolla, M. A. Fortuna, A. Pascual-García, A. Ferrera, B. Luque, and J. Bascompte, "The architecture of mutualistic networks minimizes competition and increases biodiversity," Nature, vol. 458, no. 7241, pp. 1018-1020, 2009.

[6] A. James, J. W. Pitchford, and M. J. Plank, "Disentangling nestedness from models of ecological complexity," Nature, vol. 487, no. 7406, p. 227, 2012.

[7] R. P. Rohr, S. Saavedra, and J. Bascompte, "On the structural stability of mutualistic systems," Science, vol. 345, no. 6195, p. 1253497, 2014.

[8] A. Tacchella, D. Mazzilli, and L. Pietronero, "A dynamical systems approach to gross domestic product forecasting," Nature Physics, vol. 14, no. 8, p. 861, 2018.

[9] C. A. Hidalgo, "From useless to keystone," Nature Physics, vol. 14, no. 1, p. 9, 2018.

[10] K. Soramäki, M. L. Bech, J. Arnold, R. J. Glass, and W. E. Beyeler, "The topology of interbank payment flows," Physica A: Statistical Mechanics and Its Applications, vol. 379, no. 1, pp. 317-333, 2007.

[11] S. Bustos, C. Gomez, R. Hausmann, and C. A. Hidalgo, "The dynamics of nestedness predicts the evolution of industrial ecosystems," PloS One, vol. 7, Article ID e49393, 2012.

[12] B. Jordi, J. Pedro, C. J. Melián, and J. M. Olesen, "The nested assembly of plant-animal mutualistic networks," Proceedings of the National Academy of Ences of the United States of America, vol. 100, pp. 9383-9387, 2003.

[13] N. J. Gotelli and W. Ulrich, "Statistical challenges in null model analysis," Oikos, vol. 121, no. 2, pp. 171-180, 2012.

[14] S. Saavedra, F. Reed-Tsochas, and B. Uzzi, "A simple model of bipartite cooperation for ecological and organizational networks," Nature, vol. 457, no. 7228, pp. 463-466, 2009.

[15] G. Strona, D. Nappo, F. Boccacci, S. Fattorini, and J. Sanmiguelayanz, "A fast and unbiased procedure to randomize ecological binary matrices with fixed row and column totals," Nature Communications, vol. 5, p. 4114, 2014.

[16] B. K. Fosdick, D. B. Larremore, J. Nishimura, and J. Ugander, "Configuring random graph models with fixed degree sequences," Siam Review, vol. 60, no. 2, pp. 315-355, 2018.

[17] C. Orsini, M. M. Dankulov, P. Colomerdesimon et al., "Quantifying randomness in real networks," Nature Communications, vol. 6, p. 8627, 2015.

[18] B. G. Jonsson, "A null model for randomization tests of nestedness in species assemblages," Oecologia, vol. 127, no. 3, pp. 309-313, 2001.

[19] C. Payratoborras, L. Hernandez, and Y. Moreno, "Breaking the spell of nestedness: the entropic origin of nestedness in mutualistic systems," Physical Review X, vol. 9, 2019.

[20] M. A. Fortuna, D. B. Stouffer, J. M. Olesen et al., "Nestedness versus modularity in ecological networks: two sides of the same coin?" Journal of Animal Ecology, vol. 79, pp. 811-817, 2010.

[21] A. Sole-Ribalta, C. J. Tessone, M. S. Mariani, and J. Borgeholthoefer, "Revealing in-block nestedness: detection and benchmarking," Physical Review E, vol. 97, Article ID 062302, 2018.

[22] S. H. Lee, "Network nestedness as generalized core-periphery structures," Physical Review E, vol. 93, Article ID 022306, 2016.

[23] S. Jonhson, V. Dominguezgarcia, and M. A. Munoz, "Factors determining nestedness in complex networks," PLoS One, vol. 8, 2013. 
[24] R. Hausmann and C. A. Hidalgo, "The network structure of economic output," Journal of Economic Growth, vol. 16, no. 4, pp. 309-342, 2011.

[25] T. Squartini, G. Caldarelli, G. Cimini, A. Gabrielli, and D. Garlaschelli, "Reconstruction methods for networks: the case of economic and financial systems," Physics Reports, vol. 757, pp. 1-48, 2018.

[26] L. Ermann and D. L. Shepelyansky, "Ecological analysis of world trade," Physics Letters A, vol. 377, no. 3-4, pp. 250-256, 2013.

[27] L. G. A. Alves, G. Mangioni, I. Cingolani, F. A. Rodrigues, P. Panzarasa, and Y. Moreno, "The nested structural organization of the worldwide trade multi-layer network," Scientific Reports, vol. 9, p. 2866, 2019.

[28] T. Squartini, G. Fagiolo, and D. Garlaschelli, "Randomizing world trade. i. a binary network analysis," Physical Review E, vol. 84, Article ID 046117, 2011.

[29] T. Squartini, G. Fagiolo, and D. Garlaschelli, "Randomizing world trade. ii. a weighted network analysis," Physical Review E, vol. 84, Article ID 046118, 2011.

[30] F. Saracco, R. D. Clemente, A. Gabrielli, and T. Squartini, "Randomizing bipartite networks: the case of the world trade web,” Scientific Reports, vol. 5, p. 10595, 2015.

[31] M. Barigozzi, G. Fagiolo, and D. Garlaschelli, "Multinetwork of international trade: a commodity-specific analysis," Physical Review E, vol. 81, Article ID 046104, 2010.

[32] Z.-M. Ren, A. Zeng, and Y.-C. Zhang, "Bridging nestedness and economic complexity in multilayer world trade networks," Humanities and Social Sciences Communications, vol. 7, Article ID 156, 2020.

[33] W. Atmar and B. D. Patterson, "The measure of order and disorder in the distribution of species in fragmented habitat," Oecologia, vol. 96, no. 3, pp. 373-382, 1993.

[34] B. Balassa, "Trade liberalisation and "revealed" comparative advantage," The Manchester School, vol. 33, no. 2, pp. 99-123, 1965.

[35] L. De Benedictis and L. Tajoli, "The world trade network," The World Economy, vol. 34, no. 8, pp. 1417-1454, 2011.

[36] A. Fronczak and P. Fronczak, "Statistical mechanics of the international trade network," Physical Review E, vol. 85, Article ID 056113, 2012.

[37] M. G. Kendall, Rank Correlation Methods, American Psychological Association, Washington, DC, USA, 1948.

[38] S. J. Beckett, C. A. Boulton, and H. T. P. Williams, "Falcon: a software package for analysis of nestedness in bipartite networks," F1000Research, vol. 3, p. 185, 2014.

[39] M. E. J. Newman, "Mixing patterns in networks," Physical Review E, vol. 67, Article ID 026126, 2003.

[40] J. G. Foster, D. V. Foster, P. Grassberger, and M. Paczuski, "Edge direction and the structure of networks," Proceedings of the National Academy of Sciences, vol. 107, no. 24, pp. 10815-10820, 2010.

[41] A. Grimm and C. J. Tessone, "Analysing the sensitivity of nestedness detection methods," Applied Network Science, vol. 2, p. 37, 2017.

[42] J. Borgeholthoefer, R. A. Banos, C. Gracialazaro, and Y. Moreno, "Emergence of consensus as a modular-to-nested transition in communication dynamics," Scientific Reports, vol. 7, p. 41673, 2017. 